\title{
Complexity of patients with chronic obstructive pulmonary disease hospitalized in internal medicine: a survey by FADOI
}

\author{
Carlo Nozzoli, ${ }^{1}$ Luigi Anastasio, ${ }^{2}$ Leonardo M. Fabbri, ${ }^{3}$ Pietro Marino, ${ }^{4}$ Roberto Nardi,${ }^{5}$ Antonio Sacchetta,${ }^{6}$ \\ Franco Mastroianni, ${ }^{7}$ Giovanni Mangano, ${ }^{8}$ Fabrizio Lombardini, ${ }^{9}$ Angela Zappaterra, ${ }^{10}$ Antonella Valerio, ${ }^{11}$ \\ Giorgio Vescovo, ${ }^{12}$ Giancarlo Agnelli, ${ }^{13}$ Mauro Campanini, ${ }^{14}$ for the Research Department of FADOI
}

\begin{abstract}
${ }^{1}$ Department of Internal Medicine, AOU Careggi, Florence; ${ }^{2}$ Internal Medicine, Jazzolino Hospital, Vibo Valentia; ${ }^{3}$ Oncology Haematology and Respiratory Diseases, University of Modena and Reggio Emilia, Modena; ${ }^{4}$ Emergency Medicine, Fatebenefratelli Hospital, Milan; ${ }^{5}$ Internal Medicine C, Maggiore Hospital, Bologna; ${ }^{6}$ Internal Medicine, Hospital of Conegliano Veneto (TV); ${ }^{7}$ Internal Medicine, F. Miulli Hospital, Acquaviva delle Fonti (BA); ${ }^{8}$ Internal Medicine, Fatebenefratelli Hospital, Palermo; ${ }^{9}$ Internal Medicine, S.S. Trinità Hospital, Cagliari; ${ }^{10}$ Internal Medicine, S. Chiara Hospital, Trento; ${ }^{11}$ Research Department, FADOI Foundation, Milan; ${ }^{12}$ Internal Medicine, San Bortolo Hospital, Vicenza; ${ }^{13}$ Internal and Cardiovascular Medicine and Stroke Unit, University of Perugia, Perugia; ${ }^{14}$ Department of Internal Medicine, Maggiore della Carità Hospital, Novara, Italy
\end{abstract}

\begin{abstract}
Chronic obstructive pulmonary disease (COPD) is one of the most frequent pathologies among patients hospitalized in Internal Medicine (IM) Departments. COPD is frequently associated with concomitant diseases, which represent major causes of death, and affect disease management. Objectives of our study are to assess the prevalence of COPD patients in IM, to evaluate their comorbidity status, and to describe their complexity, by means of the validated multidimensional prognostic index (MPI) score. COMPLEXICO is an observational, prospective, multicenter study, enrolling consecutive patients hospitalized for any cause in IM, with diagnosis of COPD documented by spirometry. A total of 1002 patients in 43 IM Units in Italy were enrolled. The prevalence of COPD in IM was found to be $18.1 \%$, and $72.8 \%$ of patients had at least three chronic diseases other than COPD. The mean MPI was $0.43 \pm 0.15$, and according to a stratification algorithm $31.8 \%$ of patients were classified as having low-risk, $58.9 \%$ moderate-risk and $9.3 \%$ severe-risk of adverse outcome. More than two-thirds of COPD patients

Correspondence: Roberto Nardi, via C. Pavese 16/2, 40141 Bologna, Italy.

Tel.: +39.335.8291342. E-mail: nardidoc48@gmail.com in our study present moderate to severe risk of poor outcome according to the MPI stratification.
\end{abstract}

Key words: Internal medicine; complexity; chronic obstructive pulmonary disease; multidimensional prognostic index.

Funding/support: the study was supported by an unrestricted research grant by Boehringer Ingelheim Italy, without involvement in the design and conduct of the study; collection, management, analysis, and interpretation of the data; and preparation, review, or approval of the manuscript; and decision to submit the manuscript for publication.

Acknowledgments: we are indebted to Irene Zaratti, Davide Ghilardi (Research Department of FADOI) for data management and organization.

Conflict of interest: the authors declare no conflict of interest.

See online Appendix for Members of the FADOI-COMPLEXICO Study Group.

Received for publication: 21 May 2014.

Revision received: 29 July 2014.

Accepted for publication: 29 July 2014.

This work is licensed under a Creative Commons Attribution NonCommercial 3.0 License (CC BY-NC 3.0).

(C) Copyright C. Nozzoli et al., 2015

Licensee PAGEPress, Italy

Italian Journal of Medicine 2015; 9:120-124

doi:10.4081/itjm.2015.516

\section{Introduction}

Chronic obstructive pulmonary disease (COPD) is the most frequent chronic pulmonary disease, with a reported prevalence of $20 \%$ in the elderly (above 70 -years old). ${ }^{1,2}$ All over the world, COPD is responsible for 4 death/min and the World Health Organization foresees that it will be the third cause of death by 2020 , after cardiovascular diseases and cancer. ${ }^{3}$ In Italy it is estimated that there are about 6 millions subjects affected by COPD, which is one of the first reasons for hospitalization and causes about 20,000 deaths per year, with enormous social and human costs.

COPD is primary known as a respiratory disease, and it causes invalidating outcomes per se, but a further important issue is its association with comorbidities that require medical attention and that have a higher prevalence in COPD patients than in nonCOPD ones (cardiovascular diseases, metabolic syndrome, osteoporosis, diabetes, depression, cancer, etc..$^{4}$ These comorbidities might be randomly associated with COPD (due to common risk factors includ- 
ing age), but many data support the hypothesis that chronic inflammation derived from airway wall and lung parenchyma of COPD patients may spill over the systemic circulation and mediate, at least partially, negative effects on other organs or systems. Further, some comorbidities seem to be associated with the functional abnormalities of COPD (like skeletal muscle dysfunction or osteoporosis, which are related to the inactivity due to dyspnea).

As a matter of fact, comorbidities represent major causes of death in COPD patients, they are responsible for poorer quality of life and affect disease management during COPD exacerbations. This scenario therefore claims for a comprehensive approach for COPD patients, including specific strategies able to face the several pulmonary and extra-pulmonary components of the disease.

In view of the above, the chronic comorbidities of COPD should be carefully searched for and evaluated in order to allow an appropriate framework for diagnosis and prognosis of patients with COPD. Beyond that, however, it is probably important a more careful characterization of the patient with COPD in function of its complexity, meaning not only the presence of concomitant diseases, but also a better definition of social support available to the patient, as well as of his/her cognitive and functional status, adherence to treatment, the risk of adverse reactions, etc. ${ }^{5}$ These latter, which are not generally considered in both clinical assessment and indications from guidelines, could be of particular relevance in patients with COPD who require life-long assistance and adherence to therapies.

COPD is one of the most frequent diseases in patients hospitalized in Internal Medicine (IM) Units. Two recent surveys performed by our Scientific Society reported prevalence of $15 \%$ to more than $20 \% .{ }^{6,7}$ Moreover, the very majority of patients hospitalized in IM is elderly, frail, and characterized by multiple concomitant diseases and therapies. The setting of Internal Medicine seems therefore particularly adequate to evaluate the real-life burden of comorbidities and complexity in COPD, as well as the diagnostic and therapeutic approach, and the risk profile of this patient population.

\section{Materials and Methods}

COMPLEXICO is an observational, prospective, multicenter study involving 43 Internal Medicine Units throughout Italy, sponsored by the Italian Federation of Associations of Hospital Doctors on Internal Medicine (FADOI), and coordinated by the FADOI Foundation Clinical Research Department (Figure 1).

The enrollment of patients was from February to November 2012, and patients had to be evaluated during their hospital stay.

Consecutive patients hospitalized for any cause in
Internal Medicine Units with known diagnosis of COPD documented in the past, or assessed during or shortly after the index hospitalization, by means of spirometry, were included in the study. Informed consent for the use of health data was collected for each patient prior to enrollment.

Patients were excluded if they have had severe impairment of their clinical or cognitive status, and therefore not able to comply with the procedures of data recording foreseen by the study. For patients hospitalized in critical conditions, data recording for the study could be made during hospital stay, after reaching clinical stability.

To protect the privacy, patients have been registered using a numeric code. No patient's initials, nor date of birth, or other demographic information from which it was possible to trace the identity of the subjects have been recorded.

The research was conducted in accordance with the existing rules, and approved by the Ethics Committees of the participating Centers.

The following information was collected for each patient: i) age, gender, body mass index; ii) length of hospitalization; iii) routine blood exams; iv) instrumental exams possibly made during the hospitalization; v) treatments for COPD during hospital stay (drugs, oxygen therapy, etc.); vi) other drug treatments at admission, performed during hospitalization, and prescribed at discharge; vii) COPD diagnosis (new or known); viii) COPD signs and symptoms; ix) GOLD modified questionnaire; $\mathrm{x}$ ) concomitant (acute or chronic) diseases and other conditions having clinical significance (smoke, obesity, metabolic syndrome, physical activity, etc.); xi) assessment of the complexity of the patient by means of the multidimensional prognostic index (MPI) score, a prognostic index for 1-year mortality which combines the basic and instrumental activities of daily living, level of comorbidity, cognitive status, nutritional status, the risk of pressure sores, social status, the number of drugs taken.

For the purpose of definition of the MPI score, the following questionnaire and domains were evaluated: activities of daily living (ADL) index, instrumental activities of daily living (IADL) scale, cumulative illness rating scale (CIRS), short portable mental status questionnaire (SPMSQ), mini nutritional assessment (MNA), the exton-smith scale (ESS), living (alone, family, nursing home) and number of drugs taken.

With regard to the MPI score, the Investigators collected the data for each item that contributes to the definition of the index, while the calculation of the latter has been carried out by the Promoter, and subsequently communicated to the research Centers.

\section{Statistical analysis}

The demographic and clinical-pathological characteristics were summarized by descriptive statistics. In 
general, absolute frequencies and percentages are used for qualitative variables, while mean, standard deviation (SD), minimal and maximal value (range), and median are calculated for the quantitative variables.

Due to the exploratory and descriptive aims of the study, no formal definition of the sample size was made. Based on study feasibility, an enrollment of 1000 patients was planned.

\section{Results}

A total of 1002 patients with COPD diagnosis, documented by spirometry, have been enrolled in the COMPLEXICO study.

The (seasonal) prevalence of COPD among patients hospitalized in IM and according to study inclusion criteria, was $18.1 \%$.

General characteristics of patients included in the study are described in Table 1; mean age of the study population was around 75, and around two thirds of the patients with known diagnosis had acute exacerbation of COPD at the time of the index hospitaliza-
Table 1. General characteristics of the study population.

General characteristics of the study population $(\mathrm{n}=1002 \mathrm{pts})$

\begin{tabular}{ll}
\hline Age $($ mean \pm SD) & $74.4 \pm 4.9$ \\
\hline Male gender (\%) & 69.5 \\
\hline BMI (mean \pm SD) & $26.9 \pm 0.8$ \\
\hline Smoking $(\%)$ & \\
Active & 25.8 \\
Former & 56.2 \\
No & 18.0 \\
\hline
\end{tabular}

Living (\%)

Alone $\quad 15.6$

Family $\quad 83.0$

Nursing home $\quad 1.4$

COPD (\%)

Known diagnosis $\quad 86.7$

Exacerbations $\quad 62.6$

New diagnosis during hospitalization $\quad 13.3$

SD, standard deviation; BMI, body mass index; COPD, chronic obstructive pulmonary disease.

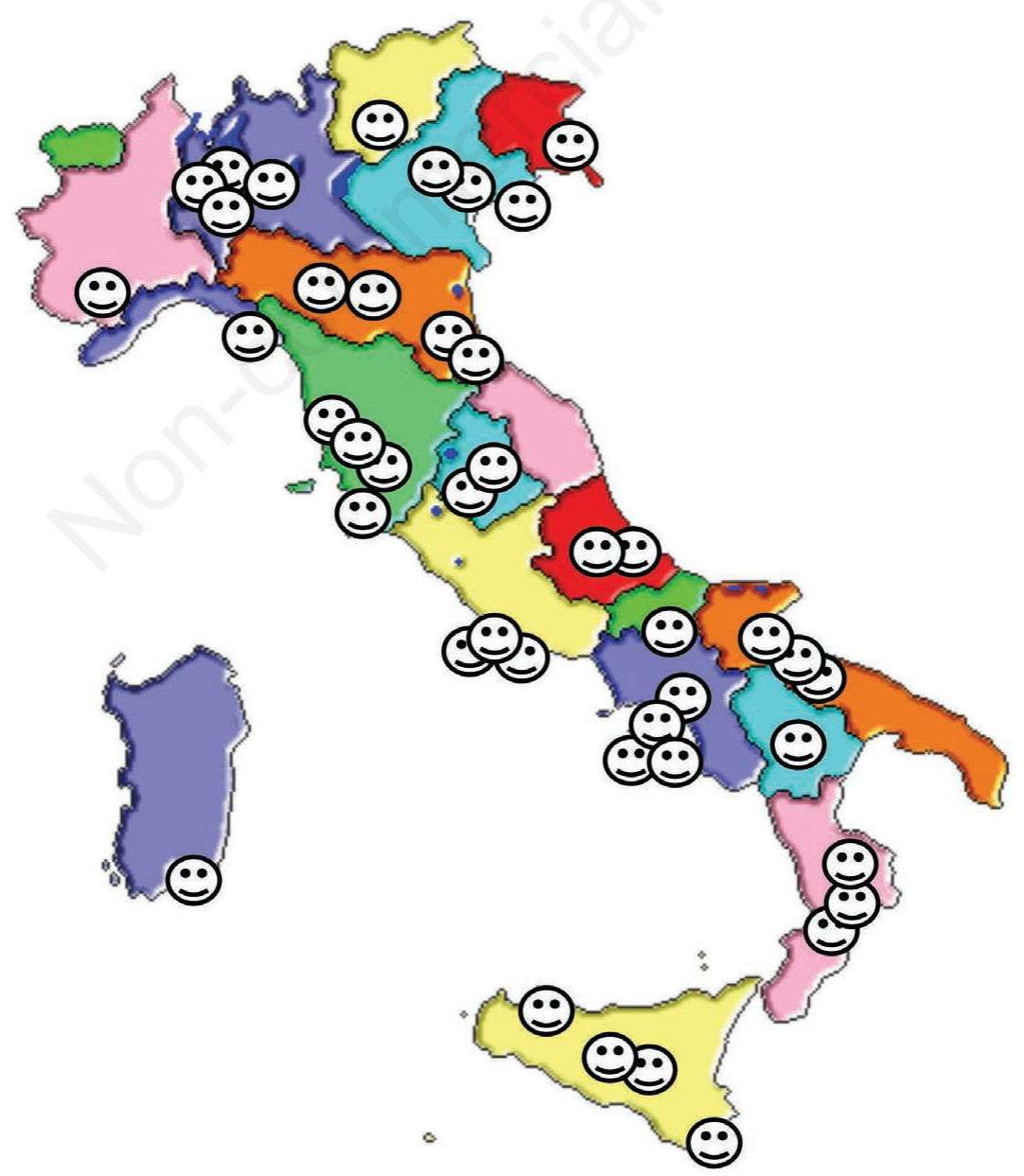

Figure 1. Geographical distribution of the 43 participating Centers. 
tion. A new diagnosis of COPD was made in $13.3 \%$ of the study population.

For patients with de novo diagnosis of COPD at the time of enrollment for the COMPLEXICO study, $91.9 \%$ underwent spirometry during hospital stay, and $8.1 \%$ after discharge. In the subgroup of patients with a previous diagnosis of COPD, spirometry was performed before, during or after hospitalization in $45.2 \%, 49.5 \%$ and $5.3 \%$ of patients, respectively.

Around three out of four patients in our study population $(72.8 \%)$ had at least three concomitant chronic diseases other than COPD. The mean number of diagnoses $( \pm \mathrm{SD})$ other than COPD assessed during hospitalization was $4.05 \pm 2.1$. At the time of admission to hospital, the number of drugs the patients were receiving (mean $\pm \mathrm{SD}$ ) was $5.5 \pm 2.8$.

Patients enrolled in COMPLEXICO were stratified according to stages stated by the most recent version GOLD guidelines at the time of study enrollment (Figure 2).

The mean MPI score was $0.43 \pm 0.15$, and according to the stratification algorithm for calculation of this index, $31.8 \%$ of patients were classified as having low-risk, $58.9 \%$ moderate-risk and $9.3 \%$ severe-risk for one-year mortality (Figure 3).

\section{Discussion and Conclusions}

Internal Medicine patients are mostly elderly and they have multiple comorbidities, which are usually chronic, rather than self-limiting or acute diseases. Within the group of patients with COPD, the critical role of concomitant diseases for the management and prognosis, has been recently acknowledged and underlined by the GOLD guidelines. ${ }^{8}$

However, neither administrative indicators nor comorbidity indexes are probably able to completely define the complexity of elderly patients with multiple diseases and treatments, and to allow physicians to manage them more properly. For the complex patients

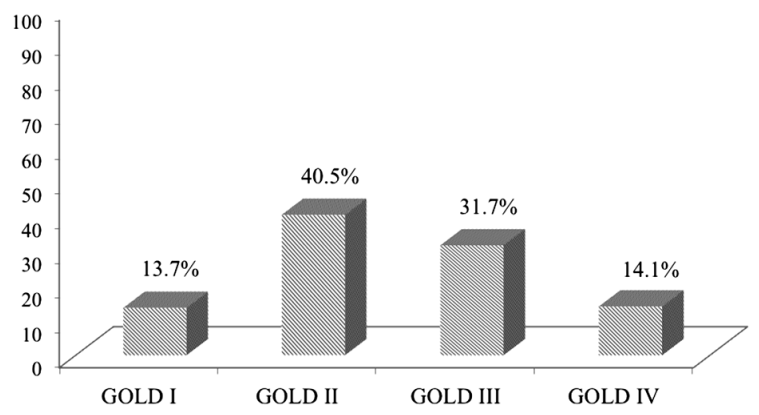

Figure 2. GOLD stratification. admitted to IM wards, internists not only need to find the best diagnosis and treatment, but also to apply a comprehensive assessment and both continuous and multidisciplinary care. This will promote good health of patients and their ability to function, and prevent or delay disability, frailty, and displacement from home and community. ${ }^{9}$ This task is particularly relevant and challenging for patients with COPD, which are very frequent among those hospitalized in IM and for whom the role of both physical and cognitive functions, as well as of social environment, are critical for the success of care.

A number of validated multidimensional tools is available to evaluate the complexity of patients. Two of them, and recently proposed, are the PROFUND and the MPI indexes. ${ }^{10,11}$ These tools, though rarely used in clinical practice, since time required for the assessment of patients is quite long, provide an accurate method of stratifying 1-year death risk in polypathological patients, both considering the overall IM or geriatrics populations, or patients selected on the basis of a specific index disease (heart failure, pneumonia, dementia, etc.) The aim of our study has been to characterize the complexity of patients with COPD confirmed by spirometry and hospitalized in IM, by applying the MPI score.

As a first finding from our study, it is confirmed that COPD is highly prevalent among patients hospitalized in Internal Medicine for any cause (18.5\% of cases had diagnosis of COPD confirmed by spirometry). Further, they have a great burden of comorbidity, since around three out of four patients in our study population had at least three diseases other than COPD at the time of their hospital stay. This percentage has been much higher than expected on the basis of previous studies in the general population of patients hospitalized in IM: ${ }^{6,7}$ this might be explained by a significant underreporting in previous surveys which were not focused on this topic, as well as by a specific relevant burden of comorbidity in the setting of COPD patients.

Around $45 \%$ of patients in the COMPLEXICO

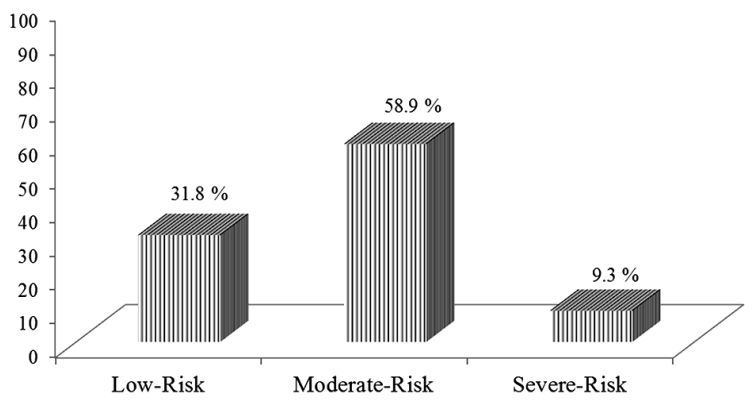

Figure 3. Stratification of risk according to multidimensional prognostic index score. 
study population were classified as GOLD stage 3 or 4 , and around two thirds of COPD patients had acute exacerbation at the time of hospital stay. These figures account for a patient profile and present clinical condition of not negligible severity, coherent with the attitude to hospitalize COPD patients mainly when acutely and critically ill.

The mean MPI score for our study population was $0.43 \pm 0.15$, a value very similar to those observed in two previous studies in geriatric patients with pneumonia $(0.43 \pm 0.25)^{12}$ and in a group of older patients with heart failure $(0.44 \pm 0.20) .{ }^{13}$ According to the predictive value of MPI score, $58.9 \%$ and $9.3 \%$ of patients were considered at moderate and severe risk of one-year mortality, respectively. These percentages seem to further underline that patients with COPD and hospitalized in IM for acute exacerbation or concomitant illnesses, represent a subpopulation with challenging clinical conditions and need for care.

In conclusion, data from the FADOI-COMPLEXICO study confirm that COPD patients hospitalized in Internal Medicine wards have a relevant level of comorbidity, and more than two-thirds of patients appear at moderate to severe prognosis if stratified according to the validated MPI score. The COMPLEXICO study may help to raise awareness of physicians on the complexity of COPD patients and the opportunity to consider a more comprehensive diagnostic and therapeutic approach for their management.

\section{References}

1. Global Global Initiative for Chronic Obstructive Lung Disease (GOLD). Global strategy for the diagnosis, management and prevention of COPD. Available from: http://www.goldcopd.org/ Accessed: April 16, 2010.

2. Niewohener DE. Clinical practice. Outpatient manage- ment of severe COPD. N Engl J Med 2010;362:1407-16.

3. Lopez AD, Shibuya K, Rao C, et al. Chronic obstructive pulmonary disease current burden and future projections. Eur Resp J 2006;27:397-412.

4. Crockett AJ, Price D. Co-morbid disease in COPD more than a coincidence. Int J COPD 2007;2:399-401.

5. Nardi R, Scanelli G, Corrao S, et al. Co-morbidity does not reflect complexity in internal medicine patients. Eur J Intern Med 2007;18:359-68.

6. Gussoni G, Campanini M, Silingardi M, et al. In-hospital symptomatic venous thromboembolism and antithrombotic prophylaxis in Internal Medicine. Thromb Haemost 2009;101:893-901.

7. Civardi G, Zucco F, Valerio A, et al. Pain management in Internal Medicine and effects of a standardized educational intervention: the FADOI-DOMINO study. Int J Clin Pract 2015;69:33-40.

8. Vestbo J, Hurd SS, Agusti AG, et al. Global strategy for diagnosis, management, and prevention of chronic obstructive pulmonary disease: GOLD evecutive summary. Am J Respir Crit Care Med 2013;187:347-65.

9. Nardi R, Berti F, Greco A, et al. Complexity in hospital internal medicine departments: what are we talking about? Ital J Med 2013;7:142-55.

10. Pilotto A, Ferrucci L, Franceschi M, et al. Development and validation of a multidimensional prognostic index for one-yar mortality from comprehensive geriatric assessment in hospitalized older patients. Rejuvenation Res 2008;11:151-61.

11. Bernabeu-Wittel M, Ollero-Baturone M, MorenoGavino L, et al. Development of a new predictive model for polypathological patients. The PROFUND index. Eur J Intern Med 2011;22:311-7.

12. Pilotto A, Addante, Ferrucci L, et al. The multidimensional prognostic index predicts short- and long-term mortality in hospitalized geriatric patients with pneumonia. J Gerontol A Biol Sci Med Sci 2009;64:880-7.

13. Pilotto A, Addante F, Franceschi M, et al. Multidimensional prognostic index based on a comprehensive geriatric assessment predicts short-term mortality in patients with heart failure. Circ Heart Fail 2010;3:14-20. 